\title{
Effects of cisapride on QTc interval in neonates
}

\author{
S Bernardini, D S Semama, F Huet, C Sgro, J B Gouyon
}

\begin{abstract}
Aim-Prospective survey of the effects of cisapride on QTc interval in neonates given cisapride.

Methods-QTc interval was determined just before and $2.9(0.9)$ days after outset of the treatment in 49 neonates treated with cisapride between 1 August 1995 and 29 February 1996.

Results-Cisapride significantly increased QTc interval $(p=0.0001)$, and this was higher when birthweight or gestational age were lower. The prolongation of QTc interval above the arbitrary value of $0.450(n=7)$ was clinically asymptomatic and was significantly more common in the infants born with a gestational age $\leqslant 33$ weeks $(\mathrm{n}=6)$.

Conclusion-The findings indicate that cisapride accumulates in less mature neonates. Further pharmacokinetic studies are needed.

(Arch Dis Child 1997;77:F241-F243)
\end{abstract}

Keywords: cisapride; QT interval; QTc interval

Cisapride is a prokinetic agent used in adults' and children's gastrointestinal motility disorders. Despite the lack of pharmacokinetic studies, this drug is widely used in neonates. The currently recommended dose in neonates and infants is 0.15 to $0.3 \mathrm{mg} / \mathrm{kg}$ three to four times a day..$^{1-3}$ Although cisapride is regarded as a safe and well tolerated drug, increased heart rate corrected QT (QTc) interval has been reported in adults ${ }^{4}$ and in one neonate ${ }^{6}$ treated with cisapride.

Similarly, we recently observed a neonate with a prolongation in QTc interval induced by cisapride. This prompted us to conduct a prospective survey to determine the prevalence of such an occurrence.

Centre Hospitalier Universitaire, 10 Bd Maréchal de Lattre de Tassigny, 21034 Dijon, Cedex, France

Service de Pédiatrie 2, Hôpital d'Enfants

$S$ Bernardini

D S Semama

F Huet

J B Gouyon

Centre Régional de Pharmacovigilance, C Sgro

Correspondence to: Dr J B Gouyon.

Accepted 11 June 1997 be prescribed. The ECG showed a prolonged QTc interval (0.490) calculated by the Bazett formula.

Serum potassium and serum calcium concentrations were within the normal range and a cerebral computed tomogram did not reveal any intracranial haemorrhage. The evoked audiometry response was normal. There was no family history of syncope, sudden death, or deafness.

The QTc interval prolongation was confirmed by two successive ECGs performed on days $32(0.489)$ and $36(0.500)$. Cisapride was subsequently withdrawn on day 40 . This was followed by a decrease in QTc interval to 0.414 by day 43 and to 0.419 by day 45 . The reintroduction of cisapride on day 48 was associated with an increase in QTc interval two days later (0.476). Cisapride treatment was stopped on day 50 and QTc interval returned to normal values on day $54(0.389)$ and remained in the normal range at 4 months of life (0.402).

This case strongly suggested that cisapride could induce asymptomatic QTc interval prolongation in premature neonates. We therefore decided to observe prospectively the effects of cisapride on QTc interval in all neonates given the drug in our department.

\section{Methods}

From 1 August 1995 to 29 February 1996 all neonates treated with cisapride were included in this open survey. For each infant, birthweight, gestational age, postnatal age, postconceptional age, weight on the day of start of treatment and associated treatments were recorded. Exclusion criteria for QTc interval analysis were: increased QTc interval (> 0.450) before cisapride administration or recognised conditions associated with an increase in QTc interval, ${ }^{7}$ such as hypocalcaemia, hypokalaemia, hypothyroidism, intracranial disorders and certain drugs, ${ }^{78}$ or those contraindicated during treatment with cisapride (ketoconazole, fluconazole, itraconazole, miconazole, erythromycin, clarithromycin, or troleandomycin). ${ }^{9}$

The ECGs were performed just before and 3 days after starting treatment with cisapride, using a Hellige simpliscriptor EK 31 or a Hewlett Packard PageWriter XLs model M1705A, with a paper speed of $25 \mathrm{~mm} /$ seconds. QTc interval was calculated as:

$$
\frac{\mathrm{QT}}{\sqrt{\mathrm{RR}}}
$$

in five beats using lead II and these five values were then averaged. Each ECG was read blind by two people. A QTc interval higher than the arbitrary value of 0.450 was regarded as significant. ${ }^{10}$ 
A blood test for potassium and calcium concentrations and a cranial sonagram were available for all the infants with an increase in QTc interval above 0.450 after cisapride treatment began. Serum potassium and serum calcium concentrations below $3.5 \mathrm{mmol} / 1$ and 2.10 $\mathrm{mmol} / \mathrm{l}$, respectively, were regarded as abnormal. Congenital hypothyroidism was excluded in all infants by the determination of serum thyrotropin concentration on day 3, in accordance with the French neonatal screening programme.

Each infant acted as its own control. QTc intervals before and after cisapride were compared using the Wilcoxon signed rank test. Linear regression was used to analyse the relation between QTc interval before treatment and gestational age; changes in QTc interval $(\Delta \mathrm{QTC})$ and other variables (birthweight, weight at the start of treatment, gestational age and postconceptional age). Comparisons among subgroups were performed using the $\chi^{2}$ test or the Mann-Whitney U test, as needed. P values of $<0.05$ were considered significant. All data were expressed as means (SD).

\section{Results}

Fifty one infants were enrolled in the survey. Two infants were subsequently excluded because of intraventricular haemorrhage in one and simultaneous treatment with erythromycin in the other. The mean birthweight and gestational age were 2240 (1003) g (range 820-4200) g and 34.6 (4.8) weeks (range 25-41 weeks), respectively. The distribution of the gestational age of these infants is shown in figure 1. On the first day of cisapride treatment, mean postnatal and postconceptional ages were 12.4 (9.7) days (range 1-43 days) and 36.3 (4.9) weeks (range 26-46.1 weeks), respectively. Mean weight at the outset of treatment was 2271 (986) g (range 730-4290 g). The mean dose of cisapride was $0.84(0.17)$ $\mathrm{mg} / \mathrm{kg} /$ day (range $0.42-1.6 \mathrm{mg} / \mathrm{kg} /$ day) administered four times daily.

QTc interval was measured just before and 2.9 (0.9) days (range 2 to 6 days) after starting cisapride. QTc interval values obtained by the two readers were not significantly different (median of the variation between the two readings: $1.1 \%$; range from $-11.8 \%$ to $9.2 \%$ ). The two readings were therefore averaged for each QTc interval. There was no correlation between QTc interval before cisapride and gestational age or birthweight.

The initiation of cisapride induced a significant increase $(\mathrm{p}=0.0001)$ in QTc interval from $0.395(0.020)$ (range $0.356-0.446)$ to $0.418(0.031)$ (range 0.371-0.504).

QTc interval increased above the arbitrary value of 0.450 in seven infants treated with cisapride. These QTc interval values were: 0.465 ; $0.468 ; 0.473 ; 0.474 ; 0.475 ; 0.478$; and 0.504 . None of these infants had clinical symptoms related to QTc interval prolongation. Six of the infants had a gestational age of $\leqslant 33$ weeks (fig 1). The risk of prolonging the QTc interval above 0.450 was significantly increased in this group. Furthermore, infants with a QTc interval above 0.450 had both significantly lower

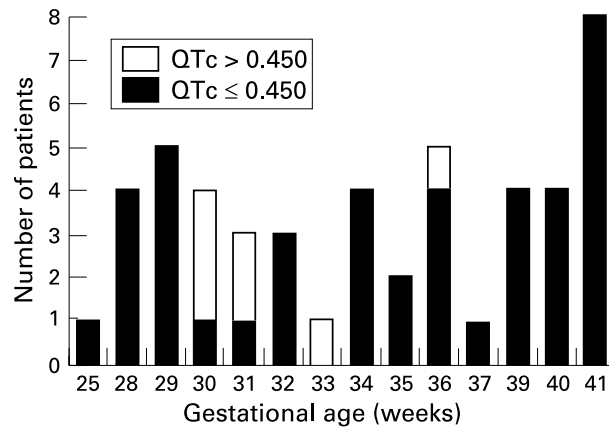

Figure 1 Distribution of neonates enrolled in the survey according to gestational age: $\square$ patients with $Q T c$ interval above 0.450 after treatment with cisapride; patients with no QTc interval increase above 0.450 after treatment with cisapride.

weight at the start of treatment and higher $\Delta$ QTc compared with the rest of the study group. There was no significant difference between these two subgroups with regard to the length of QTc interval before cisapride, birthweight, postconceptional age, postnatal age and dose of cisapride.

We also failed to find any correlation between $\Delta \mathrm{QTC}$ and the daily dose of cisapride given, postnatal age, and postconceptional age. However, $\Delta \mathrm{QT}$ c showed a weak but significant inverse correlation with gestational age $\left(\mathrm{r}^{2}=\right.$ $0.128 ; \mathrm{p}=0.012)$, birthweight $\left(\mathrm{r}^{2}=0.149 ; \mathrm{p}=\right.$ 0.006 ), weight at the beginning of cisapride treatment $\left(\mathrm{r}^{2}=0.128 ; \mathrm{p}=0.011\right)$, and QTc interval before treatment $\left(r^{2}=0,0099 ; p=\right.$ $0.028)$.

\section{Discussion}

The effects of cisapride on heart rhythm have recently come to light. The first reported effects included tachycardia. ${ }^{11-13}$ From September 1993 to April 1996, the Food and Drug Administration's MedWatch Reporting Program (spontaneous reporting system) was sent 23 cases of QT interval prolongation in patients taking cisapride. Most of those patients were chronically ill, had other potential risk factors for arrhythmias, or were receiving a cocktail of drugs. ${ }^{9}$

In 1995 Janssen Pharmaceuticals warned physicians in the USA about two cases of ventricular arrhythmias, including torsades de pointes in patients treated with both cisapride and ketoconazole. ${ }^{5}$ The same year, the first published case of increased QTc interval assigned to cisapride was reported in a 64 year old man treated with high doses of cisapride for a diabetic gastric atony. The QTc interval increase (0.550) was revealed as a result of syncope. When cisapride was gradually reduced, the QTc interval returned to normal. ${ }^{4}$

In 1996 Lewin et $a l^{6}$ reported the first cisapride induced QTc interval increase in a neonate. This infant was born at 30 weeks of gestational age and presented at 2 months of age with a 2:1 atrioventricular conduction with a prolonged QTc interval of 0.510 . He had been prescribed cisapride at a dose of 0.3 $\mathrm{mg} / \mathrm{kg}$ four times a day. Once cisapride was discontinued, the QTc interval reverted to normal. 
Our index case indicates a role for cisapride in the prolongation of QTc interval because treatment was associated with QTc interval increases which stopped once its use had been discontinued. The prolongation of QTc interval could not be ascribed to a cisapridemiconazole interaction because absorption of cutaneous application of miconazole is poor and especially because the QTc interval was still increased 10 days after miconazole had been discontinued.

Our prospective survey has confirmed and extended those limited observations. It has shown that cisapride increased QTc interval and that this increase was higher when the birthweight or gestational age were low. Moreover, a QTc interval exceeding 0.450 was observed in $14.3 \%$ of the 49 neonates treated with the drug and this was mainly observed in neonates with a gestational age of $\leqslant 33$ weeks (six out of seven neonates).

The recent demonstration of a dose dependent class III anti-arrhythmic effect of cisapride on the Purkinje fibres of rabbits, ${ }^{14}$ suggests that the increase in QTc interval with cisapride might have been due to an accumulation of the drug. Cytochrome P-450 3A4 enzyme system affects cisapride metabolism, ${ }^{9}$ so we hypothesise that the limited cytochrome P450 function reported in neonates, particularly premature infants, ${ }^{15}$ could predispose to high plasma concentrations of the drug, and/or accumulation of its active metabolites, and/or a production of toxic metabolites by modified metabolic pathways. This hypothesis is strengthened by the interaction of ketoconazole, which inhibits cytochrome P-450 3A4, ${ }^{16}{ }^{17}$ with cisapride metabolism. ${ }^{5} 9$ Moreover, the positive correlation described between the biotransformation capacity of the liver and gestational age ${ }^{18}$ could explain why the QTc interval prolongation above 0.450 was observed only in premature infants in our survey. Pharmacokinetic studies are therefore mandatory in newborn infants in order to establish a safe therapeutic dose regimen for this particular group.

1 Melis K, Janssens G. Long term use of cisapride Prepulsid $\AA$ ) in premature neonates. Acta Gastroenterol Belg 990;53:372-5.

2 Janssens G, Melis K, Vaerenberg M. Long term use of cisapride (Prepulsid $\AA$ ) in premature neonates of less 34 weeks gestational age. $\mathcal{F}$ Pediatr Gastroenterol Nutr 1990;11:420-5.

3 Wiseman LR, Faulds D. Cisapride. An update review of its pharmacology and therapeutic efficacy as a prokinetic agent in gastrointestinal motility disorders. Drugs 1994;47:116-52.

4 Bran S, Murray WA, Hirsch IB, Palmer JP. Long QT syndrome during high dose cisapride. Arch Intern Med 1995;155:765-8.

5 Ahmad SR, Wolfe SM. Cisapride and torsades de pointes. Lancet 1995;345:508.

6 Lewin MB, Bryant RM, Fenrich AL, Grifka RG. Cisaprideinduced long QT interval. F Pediatr 1996;128:279-81.

7 Tan HL, Hou CJY, Lauer MR, Sung RJ. Electrophysiologic mechanisms of the long QT interval syndromes and mechanisms of the long QT interval syndromes
torsade de pointes. Ann Intern Med 1995;122:701-14.

8 Zehender M, Hohnloser S, Just H. QT-interval prolonging drugs: Mechanisms and clinical relevance of their arrhythmogenic hazards. Cardiovasc Drugs Ther 1991;5:515-30.

9 Wysowski DK, Bacsanyi J. Cisapride and fatal arrhythmia. N Engl f Med 1996;335:290-1.

10 Schwartz PJ, Montemerlo M, Facchini M, et al. The QT interval throughout the first six months of life: A prospective study. Circulation 1982;66:496-501.

11 Olsson S, Edwards IR. Tachycardia during cisapride treatment. BMF 1992;305:748-9.

12 Usha Rani P, Naidu MUR, Ramesh-Kumar T, Shobha JC, Kumar A. Tachycardia during cisapride treatment. Indian fPharmacol 1994;26:233-4.

13 Woodard-Jenkins J, Woolf A. Spectrum of toxicity seen in cisapride poisoning. F Toxicol 1995;33:490A.

14 Puisieux FL, Adamantidis MM, Dumotier BM, Dupuis BA. Cisapride-induced prolongation of cardiac action potential and early afterdepolarizations in rabbit Purkinje fibres. Br F Pharmacol 1996;117:1377-9.

15 Smith Rogers A. The role of cytochrome P450 in developmental pharmacology. $\mathcal{f}$ Adolesc Health 1994;15:635-40

16 Greenblatt DJ, Von Moltke LL, Harmatz JS, et al. Interaction of triazolam and ketoconazole. Lancet 1995;345:191.

17 Ervine CM, Matthew DE, Brennan B, Housyon JB. Comparison of ketoconazole and fluconazole as cyto15.

18 Boehm G, Teichmann B, Krumbiegel P. Hepatic biotransformation capacity in low-birth-weight infants as measured with the $[15 \mathrm{~N}] \mathrm{Meth}$ cetin urine test: Influences of ured with the $[15 \mathrm{~N}]$ Methacetin urine test: Influences of gestational age, postnatal age, and int 